\title{
An open study of medical cannabis treatment in PTSD and PDD adult ambulatory patients
}

Post-Traumatic Stress Disorder (PTSD) and Pervasive Developmental Disorder (PDD) are relatively common disturbances. Both present a treatment challenge with disappointing outcomes and frustrating results.

I aim to present the results of open study of about thousand PTSD patients and hundred PDD patients that were treated by Medical Cannabis (MC) in my clinic since 2008 (PTSD) and 2014 (PDD).

The diagnosis was made upon DSM5 symptoms checklist.

All the patients received the treatment approval from The Israeli Ministry of Health (MoH) to smoke between 30-50 gram/m of MC (PTSD) and the same amount of Cannabis oil (PDD). The MC was supplied aleatory by Israeli Cannabis Companies from $\mathrm{MoH}$ list. The cannabis strain was selected for the personal needs of the patient, until 16\% THC for PTSD patients and based mainly on rich CBD oil for PDD patients. MC was added on their regular treatment.

The preconditions for MC approval in PTSD were two significant psychiatric treatment trials and at least one kind psychotherapy course.

For the PDD group, the diagnosis was made in childhood. The patients were psychiatric treated in the past.

The results showed a significant clinical improvement for both groups and stability during time.

The PTSD patients described a better sleep, continuous and without nightmares, less hyperarousal, anxiety, anhedonia and traumatic intrusions.

They described improvement in social and occupational functioning.

$74.5 \%$ stopped all medication. $68 \%$ returned to work/ study (29.1\% before treatment).

The PDD patients improved in violent, obsessive and behavior, showed improvement in affective and cognitive symptoms. 